\title{
Variable-coefficient symbolic computation approach for finding multiple rogue wave solutions of nonlinear system with variable coefficients
}

\author{
Jian-Guo Liu*, Wen-Hui Zhu, Yan He
}

Received: date / Accepted: date

\begin{abstract}
In this paper, a variable-coefficient symbolic computation approach is proposed to solve the multiple rogue wave solutions of nonlinear equation with variable coefficients. As an application, a $(2+1)$-dimensional variablecoefficient Kadomtsev-Petviashvili equation is investigated. The multiple rogue wave solutions are obtained and their dynamics features are shown in some $3 \mathrm{D}$ and contour plots.
\end{abstract}

Keywords variable-coefficient symbolic computation approach, rogue wave, variable coefficient Kadomtsev-Petviashvili equation.

Mathematics Subject Classification (2000) 35C08 - 68M07 · 33F10

\section{Introduction}

In this paper, the following $(2+1)$-dimensional variable-coefficient KadomtsevPetviashvili (vcKP) equation is investigated [1]

$$
\alpha(t) u_{x}^{2}+\alpha(t) u u_{x x}+\beta(t) u_{x x x x}-\gamma(t) u_{y y}+u_{x t}=0,
$$

Project supported by National Natural Science Foundation of China (Grant No 81960715).

Jian-Guo Liu $\left({ }^{*}\right.$ Corresponding author $)$, Yan He

College of Computer, Jiangxi University of Traditional Chinese Medicine, Jiangxi 330004, China

E-mail: 20101059@jxutcm.edu.cn

Jian-Guo Liu

School of science, Beijing University of Posts and Telecommunications, Beijing 100876, China

Wen-Hui Zhu

Institute of artificial intelligence, Nanchang Institute of Science and Technology, Jiangxi 330108, China

E-mail: 415422402@qq.com 
where $u=u(x, y, t)$ describes amplitude of the long wave of two-dimensional fluid domain on varying topography or in turbulent ow over a sloping bottom. $\alpha(t), \beta(t)$ and $\gamma(t)$ are arbitrary real functions. The solitonic solution [1], Wronskian and Gramian solutions [2], Bäcklund transformation [3], breather wave solutions [4], lump and interactions solutions [5, 6] of Eq. (1) have been studied.

Rogue wave has important applications in ocean's waves [7], optical fibers [8], Bose-Einstein condensates [9] and other fields. Rogue wave solutions of many integrable equations have been investigated [10-14]. Recently, a symbolic computation approach to obtain the multiple rogue wave solutions is proposed by Zhaqilao [15]. But the main application of this method is constant-coefficient integrable equation [16-18], which is not suitable for variable-coefficient integrable equation. So, we give an improved method named variable-coefficient symbolic computation approach (vcsca) to solve this problem and apply this method to Eq. (1), which will be the main work of our paper.

The organization of this paper is as follows. Section 2 proposes a vcsca; Section 3 gives the 1-rogue wave solutions; Section 4 obtains the 3-rogue wave solutions; Section 5 presents the 6-rogue wave solutions; Section 6 gives this conclusions.

\section{Modified symbolic computation approach}

Here, we present a vcsca to find the multiple rogue wave solutions of variable coefficient integrable equation

Step1. Instead of the traveling wave transformation in Ref. [15], we make a non-traveling wave transformation $v=x-\omega(t)$ in the following nonlinear system with variable coefficients

$$
\Xi\left(u, u_{t}, u_{x}, u_{y}, u_{x y}, \cdots\right)=0,
$$

Eq. (2) is reduced to a $(1+1)$-dimensional equation

$$
\Xi\left(u, u_{v}, u_{y}, u_{v y}, \cdots\right)=0 \text {. }
$$

Step2. By Painlevé analysis, we make the following transformation

$$
u(v, y)=\frac{\partial^{n}}{\partial v^{m}} \ln \xi(v, y) .
$$

$m$ can be derived by balancing the order of the highest derivative term and nonlinear term.

Step3. Assuming

$$
\xi(v, y)=F_{n+1}(v, y)+2 \nu y P_{n}(v, y)+2 \mu v Q_{n}(v, y)+\left(\mu^{2}+\nu^{2}\right) F_{n-1}(v, y),(5)
$$


with

$$
\begin{aligned}
F_{n}(v, y) & =\sum_{k=0}^{n(n+1) / 2} \sum_{i=0}^{k} a_{n(n+1)-2 k, 2 i} y^{2 i} v^{n(n+1)-2 k}, \\
P_{n}(v, y) & =\sum_{k=0}^{n(n+1) / 2} \sum_{i=0}^{k} b_{n(n+1)-2 k, 2 i} v^{2 i} y^{n(n+1)-2 k}, \\
Q_{n}(v, y) & =\sum_{k=0}^{n(n+1) / 2} \sum_{i=0}^{k} c_{n(n+1)-2 k, 2 i} y^{2 i} v^{n(n+1)-2 k},
\end{aligned}
$$

$F_{0}=1, F_{-1}=P_{0}=Q_{0}=0$, where $a_{m, l}, b_{m, l}$ and $c_{m, l}(m, l \in[0,2,4, \cdots, n(n+1)])$ are unknown constants, $\mu$ and $\nu$ are the wave center.

Step4. Substituting Eq. (4) and Eq. (5) into Eq. (3) and equating all the coefficients of the different powers of $v$ and $y$ to zero, we can know $a_{m, l}, b_{m, l}$ and $c_{m, l}(m, l \in[0,2,4, \cdots, n(n+1)])$. The corresponding multiple rogue wave solutions can be presented.

\section{1-rogue wave solutions}

Based on the vcsca, set

$$
\alpha(t)=\frac{6 \beta(t)}{\Theta_{0}}, v=x-\omega(t), u=2 \Theta_{0}[\ln \xi(v, y)]_{v v},
$$

Eq. (1) can be changed as

$$
\begin{aligned}
& 6 \xi_{v}^{2}\left[\xi\left[3 \beta(t) \xi_{v v v v}-2 \omega^{\prime}(t) \xi_{v v}\right]+3 \beta(t) \xi_{v v}^{2}\right]+2 \xi^{2} \xi_{v}\left[2 \omega^{\prime}(t) \xi_{v v v}\right. \\
- & \left.3 \beta(t) \xi_{v v v v v}\right]+\xi\left[\xi\left[-3 \beta(t) \xi_{v v v v} \xi_{v v}+2 \beta(t) \xi_{v v v}^{2}+3 \omega^{\prime}(t) \xi_{v v}^{2}\right]\right. \\
+ & \left.\xi^{2}\left[\beta(t) \xi_{v v v v v v}-\omega^{\prime}(t) \xi_{v v v v}\right]-6 \beta(t) \xi_{v v}^{3}\right]-24 \beta(t) \xi_{v v v} \xi_{v}^{3} \\
+ & \gamma(t)\left[\left[6 \xi_{v}^{2}-2 \xi \xi_{v v}\right] \xi_{y}^{2}+2 \xi\left[\xi \xi_{v v y}-4 \xi_{v} \xi_{v y}\right] \xi_{y}+\xi\left[\xi_{y y}\left[\xi \xi_{v v}-2 \xi_{v}^{2}\right]\right.\right. \\
+ & \left.\left.\xi\left[2 \xi_{v y}^{2}+2 \xi_{v} \xi_{v y y}-\xi \xi_{v v y y}\right]\right]\right]+6 \omega^{\prime}(t) \xi_{v}^{4} .
\end{aligned}
$$

According to Eq. (5), we have

$$
\xi(v, y)=(v-\mu)^{2}+\zeta_{1}(y-\nu)^{2}+\zeta_{0},
$$

where $\mu, \nu, \zeta_{0}$ and $\zeta_{1}$ are unknown real constants. Substituting Eq. (8) into Eq. (7) and equating the coefficients of all powers $v$ and $y$ to zero, we obtain

$$
\gamma(t)=\frac{3 \beta(t)}{\zeta_{0} \zeta_{1}}, \omega^{\prime}(t)=\zeta_{1} \gamma(t)
$$

Substituting Eq. (8) and Eq. (9) into Eq. (6), the 1-rogue wave solutions for Eq. (1) can be read as

$$
u=\frac{4 \Theta_{0}\left[-(\mu-v)^{2}+\zeta_{1}(y-\nu)^{2}+\zeta_{0}\right]}{\left[(\mu-v)^{2}+\zeta_{1}(y-\nu)^{2}+\zeta_{0}\right]^{2}} .
$$


When $\zeta_{0}>0$, rogue wave (10) has three extreme value points $(\mu, \nu),(\mu \pm$ $\left.\sqrt{3} \sqrt{\zeta_{0}}, \nu\right)$. When $\zeta_{0}<0, \zeta_{1}>0$, rogue wave (10) has three extreme value points $(\mu, \nu),\left(\mu, \nu \pm \frac{\sqrt{-\zeta_{0}}}{\sqrt{\zeta_{1}}}\right)$. Fig. 1 and Fig. 2 describe the dynamics features of rogue wave (10) when $\zeta_{0}$ and $\zeta_{1}$ select different values.
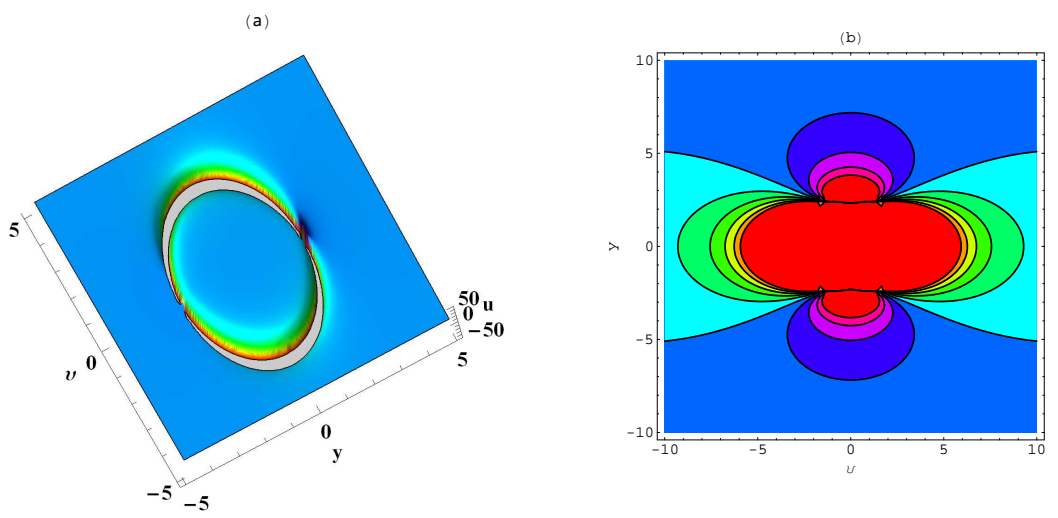

Fig. 1. Rogue wave (10) with $\mu=\nu=0, \Theta_{0}=1, \zeta_{0}=-10, \zeta_{1}=2$, (a) 3D graphic, (b) contour plot.
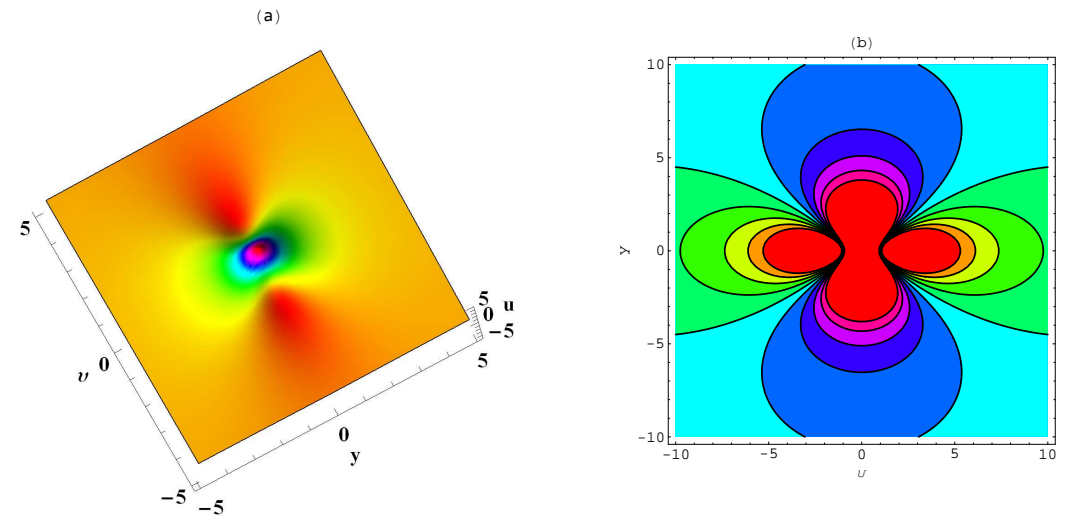

Fig. 2. Rogue wave (10) with $\mu=\nu=0, \Theta_{0}=1, \zeta_{0}=1, \zeta_{1}=2$,

(a) 3D graphic, (b) contour plot.

\section{3-rogue wave solutions}

In order to look for the 3-rogue wave solutions, we set

$$
\begin{aligned}
\xi(v, y) & =\mu^{2}+\nu^{2}+v^{6}+y^{6} \zeta_{17}+y^{4} \zeta_{16}+2 \mu v\left(y^{2} \zeta_{23}+v^{2} \zeta_{24}+\zeta_{22}\right) \\
& +2 \nu y\left(y^{2} \zeta_{20}+v^{2} \zeta_{21}+\zeta_{19}\right)+v^{4} y^{2} \zeta_{11}+y^{2} \zeta_{15} \\
& +v^{2}\left(y^{4} \zeta_{14}+y^{2} \zeta_{13}+\zeta_{12}\right)+v^{4} \zeta_{10}+\zeta_{18},
\end{aligned}
$$


where $\zeta_{i}(i=10, \cdots, 24)$ is unknown real constant. Substituting Eq. (11) into Eq. (7) and equating the coefficients of all powers $v$ and $y$ to zero, we get

$$
\begin{aligned}
\gamma(t) & =\frac{90 \beta(t)}{\zeta_{13}}, \omega^{\prime}(t)=\frac{30 \zeta_{11} \beta(t)}{\zeta_{13}}, \zeta_{14}=\frac{\zeta_{11}^{2}}{3}, \zeta_{16}=\frac{17 \zeta_{11} \zeta_{13}}{270}, \\
\zeta_{20} & =-\frac{1}{9} \zeta_{11} \zeta_{21}, \zeta_{17}=\frac{\zeta_{11}^{3}}{27}, \zeta_{15}=\frac{19 \zeta_{13}^{2}}{108 \zeta_{11}}, \zeta_{23}=-\zeta_{11} \zeta_{24}, \\
\zeta_{22} & =-\frac{\zeta_{13} \zeta_{24}}{30 \zeta_{11}}, \zeta_{12}=-\frac{5 \zeta_{13}^{2}}{36 \zeta_{11}^{2}}, \zeta_{10}=\frac{5 \zeta_{13}}{6 \zeta_{11}}, \zeta_{19}=\frac{\zeta_{13} \zeta_{21}}{18 \zeta_{11}}, \\
\zeta_{18} & =-\mu^{2}-\nu^{2}+\mu^{2} \zeta_{24}^{2}+\frac{\nu^{2} \zeta_{21}^{2}}{3 \zeta_{11}}+\frac{5 \zeta_{13}^{3}}{72 \zeta_{11}^{3}} .
\end{aligned}
$$

Substituting Eq. (11) and Eq. (12) into Eq. (6), the 3-rogue wave solutions for Eq. (1) can be read as

$$
\begin{aligned}
u & =\left[2 4 \Theta _ { 0 } \zeta _ { 1 1 } \left[5 \left[12 y^{4} \zeta_{11}^{4}+36 \zeta_{11}^{2}\left(15 v^{4}+y^{2} \zeta_{13}+2 \nu y \zeta_{21}+6 \mu v \zeta_{24}\right)\right.\right.\right. \\
& \left.+216 v^{2} y^{2} \zeta_{11}^{3}+180 v^{2} \zeta_{13} \zeta_{11}-5 \zeta_{13}^{2}\right]\left[40 y^{6} \zeta_{11}^{6}+360 v^{2} y^{4} \zeta_{11}^{5}\right. \\
& +2 \zeta_{11}^{2}\left[95 y^{2} \zeta_{13}^{2}+6 \zeta_{13}\left(75 v^{4}+10 \nu y \zeta_{21}-6 \mu v \zeta_{24}\right)+180 \nu^{2} \zeta_{21}^{2}\right] \\
& +4 y^{2} \zeta_{11}^{4}\left[y\left(17 y \zeta_{13}-60 \nu \zeta_{21}\right)+270 v\left(v^{3}-2 \mu \zeta_{24}\right)\right]+1080 \zeta_{11}^{3}\left[v^{2} y^{2} \zeta_{13}\right. \\
& \left.\left.+2 \nu v^{2} y \zeta_{21}+\left(v^{3}+\mu \zeta_{24}\right)^{2}\right]-150 v^{2} \zeta_{13}^{2} \zeta_{11}+75 \zeta_{13}^{3}\right]-12 \zeta_{11}\left[60 v y^{4} \zeta_{11}^{4}\right. \\
& +180 v \zeta_{11}^{2}\left(3 v^{4}+y^{2} \zeta_{13}+2 \nu y \zeta_{21}+3 \mu v \zeta_{24}\right)+180 y^{2} \zeta_{11}^{3}\left(2 v^{3}-\mu \zeta_{24}\right) \\
& \left.\left.\left.+6 \zeta_{13} \zeta_{11}\left(50 v^{3}-\mu \zeta_{24}\right)-25 v \zeta_{13}^{2}\right]^{2}\right]\right] /\left[\left[40 y^{6} \zeta_{11}^{6}+360 v^{2} y^{4} \zeta_{11}^{5}\right.\right. \\
& +2 \zeta_{11}^{2}\left[95 y^{2} \zeta_{13}^{2}+6 \zeta_{13}\left(75 v^{4}+10 \nu y \zeta_{21}-6 \mu v \zeta_{24}\right)+180 \nu^{2} \zeta_{21}^{2}\right] \\
& +4 y^{2} \zeta_{11}^{4}\left[y\left(17 y \zeta_{13}-60 \nu \zeta_{21}\right)+270 v\left(v^{3}-2 \mu \zeta_{24}\right)\right]+1080 \zeta_{11}^{3}\left[v^{2} y^{2} \zeta_{13}\right. \\
& \left.\left.\left.+2 \nu v^{2} y \zeta_{21}+\left(v^{3}+\mu \zeta_{24}\right)^{2}\right]-150 v^{2} \zeta_{13}^{2} \zeta_{11}+75 \zeta_{13}^{3}\right]^{2}\right],
\end{aligned}
$$

where $\zeta_{11}, \zeta_{13}, \zeta_{21}$ and $\zeta_{24}$ are unrestricted. Dynamics features of 3-rogue wave solutions are displayed in Figs. 3-6 when $(\mu, \nu)$ selects different values, we can see that three rogue waves break apart and form a set of three 1-rogue waves in Figs. 3-6.
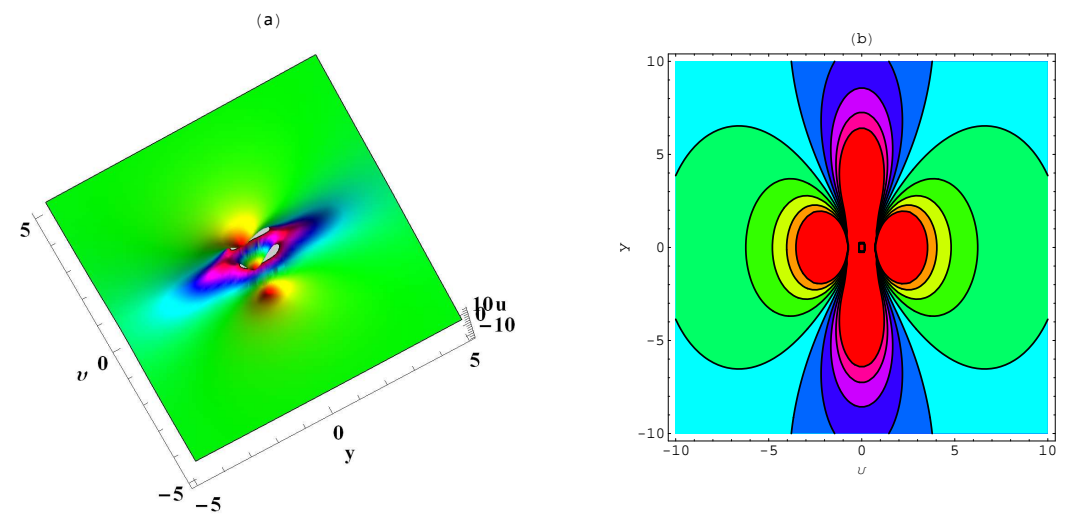
Fig. 3. Rogue wave (13) with $\mu=\nu=0, \Theta_{0}=1, \zeta_{11}=\zeta_{13}=\zeta_{21}=\zeta_{24}=1$, (a) 3D graphic, (b) contour plot.
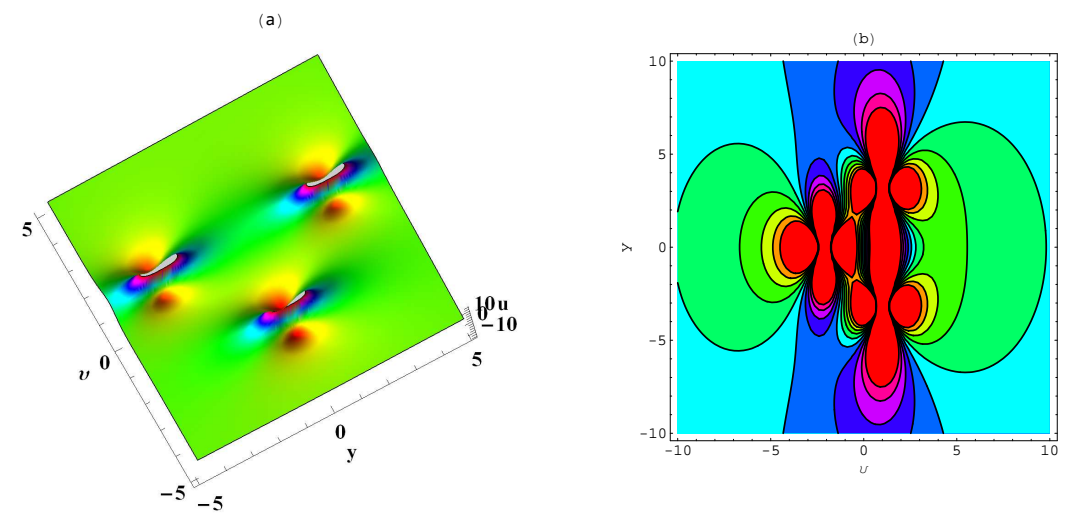

Fig. 4. Rogue wave (13) with $\mu=10, \nu=0, \zeta_{11}=\zeta_{13}=\zeta_{21}=\zeta_{24}=1$, $\Theta_{0}=1$, (a) 3D graphic, (b) contour plot.
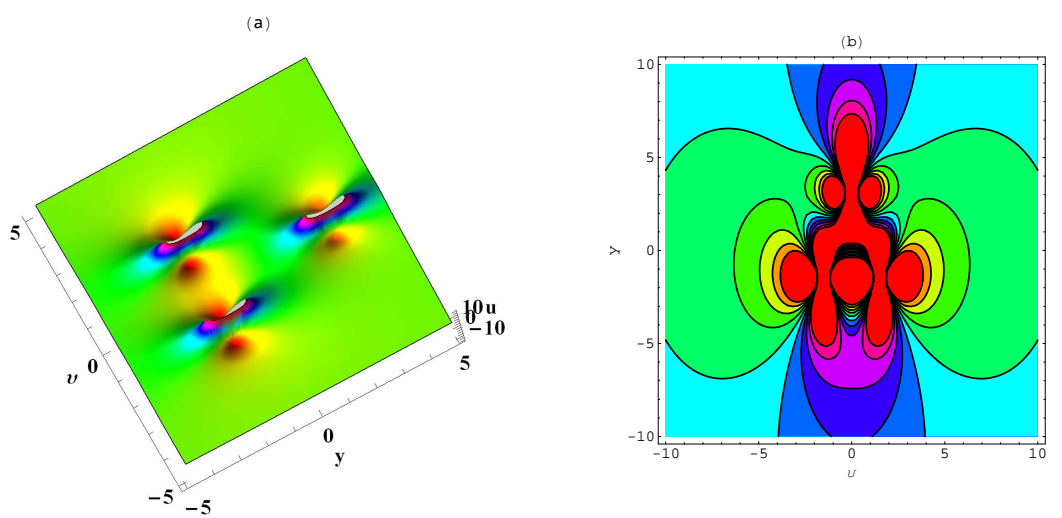

Fig. 5. Rogue wave (13) with $\mu=0, \nu=10, \zeta_{11}=\zeta_{13}=\zeta_{21}=\zeta_{24}=1$, $\Theta_{0}=1$, (a) 3D graphic, (b) contour plot.
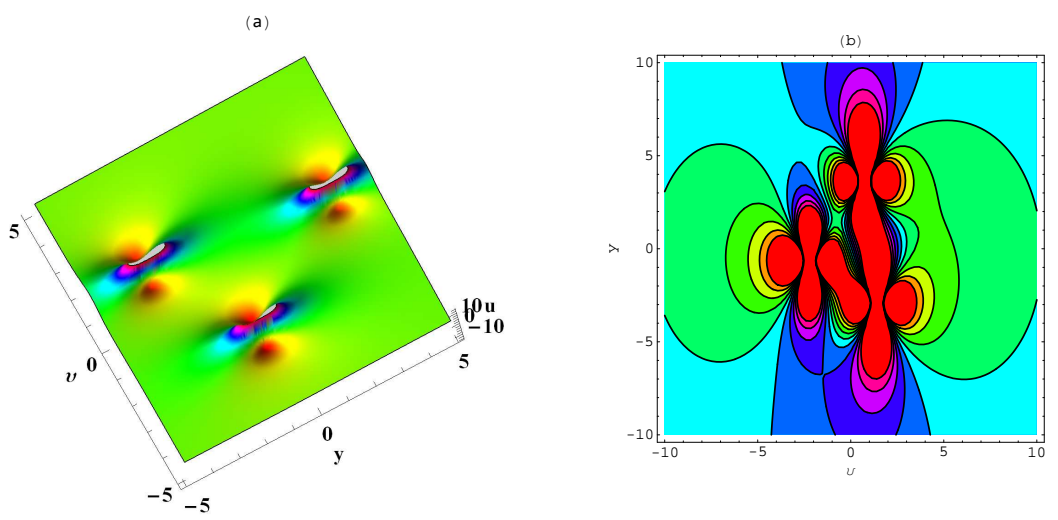
Fig. 6. Rogue wave (13) with $\mu=\nu=10, \zeta_{11}=\zeta_{13}=\zeta_{21}=\zeta_{24}=1$, $\Theta_{0}=1$, (a) 3D graphic, (b) contour plot.

\section{6-rogue wave solutions}

To present the 6-rogue wave solutions, we assume

$$
\begin{aligned}
\xi(v, y) & =v^{12}+y^{8} \zeta_{48}+y^{6} \zeta_{47}+y^{4} \zeta_{46}+v^{10}\left(y^{2} \zeta_{26}+\zeta_{25}\right) \\
& +y^{2} \zeta_{45}+v^{8}\left(y^{4} \zeta_{29}+y^{2} \zeta_{28}+\zeta_{27}\right)+2 \mu v\left[v^{6}+y^{6} \zeta_{64}+y^{4} \zeta_{63}\right. \\
& \left.+v^{4}\left(y^{2} \zeta_{69}+\zeta_{68}\right)+y^{2} \zeta_{62}+v^{2}\left(y^{4} \zeta_{67}+y^{2} \zeta_{66}+\zeta_{65}\right)+\zeta_{61}\right] \\
& +2 \nu y\left[y^{6}+y^{4}\left(v^{2} \zeta_{57}+\zeta_{56}\right)+y^{2}\left(v^{4} \zeta_{55}+v^{2} \zeta_{54}+\zeta_{53}\right)+v^{6} \zeta_{60}\right. \\
& \left.+v^{4} \zeta_{59}+v^{2} \zeta_{58}+\zeta_{52}\right]+v^{6}\left(y^{6} \zeta_{33}+y^{4} \zeta_{32}+y^{2} \zeta_{31}+\zeta_{30}\right) \\
& +v^{4}\left(y^{8} \zeta_{38}+y^{6} \zeta_{37}+y^{4} \zeta_{36}+y^{2} \zeta_{35}+\zeta_{34}\right)+v^{2}\left(y^{10} \zeta_{44}+y^{8} \zeta_{43}\right. \\
& \left.+y^{6} \zeta_{42}+y^{4} \zeta_{41}+y^{2} \zeta_{40}+\zeta_{39}\right)+\zeta_{51}+y^{12} \zeta_{50}+y^{10} \zeta_{49} \\
& +\left(\mu^{2}+\nu^{2}\right)\left[v^{2}+y^{2} \zeta_{1}+\zeta_{0}\right],
\end{aligned}
$$

where $\zeta_{i}(i=25, \cdots, 69)$ is unknown real constant. Substituting Eq. (14) into Eq. (7) and equating the coefficients of all powers $v$ and $y$ to zero, we obtain

$$
\begin{aligned}
& \gamma(t)=\frac{690 \beta(t)}{\zeta_{28}}, \omega^{\prime}(t)=\frac{1}{6} \zeta_{26} \gamma(t), \zeta_{29}=\frac{5 \zeta_{26}^{2}}{12}, \zeta_{33}=\frac{5 \zeta_{26}^{3}}{54}, \\
& \zeta_{32}=\frac{77 \zeta_{26} \zeta_{28}}{207}, \zeta_{31}=\frac{1862 \zeta_{28}^{2}}{7935 \zeta_{26}}, \zeta_{37}=\frac{73 \zeta_{26}^{2} \zeta_{28}}{1242}, \zeta_{36}=\frac{749 \zeta_{28}^{2}}{9522}, \\
& \zeta_{55}=-\frac{180}{\zeta_{26}^{2}}, \zeta_{38}=\frac{5 \zeta_{26}^{4}}{432}, \zeta_{35}=\frac{294 \zeta_{28}^{3}}{12167 \zeta_{26}^{2}}, \zeta_{43}=\frac{19 \zeta_{26}^{3} \zeta_{28}}{4968}, \\
& \zeta_{42}=\frac{77 \zeta_{26} \zeta_{28}^{2}}{6210}, \zeta_{41}=-\frac{49 \zeta_{28}^{3}}{182505 \zeta_{26}}, \zeta_{52}=\frac{271656 \zeta_{28}^{3}}{304175 \zeta_{26}^{6}} \text {, } \\
& \zeta_{54}=-\frac{1368 \zeta_{28}}{23 \zeta_{26}^{3}}, \zeta_{44}=\frac{\zeta_{26}^{5}}{1296}, \zeta_{57}=-\frac{54}{\zeta_{26}}, \zeta_{40}=\frac{3773 \zeta_{28}^{4}}{6996025 \zeta_{26}^{3}}, \\
& \zeta_{50}=\frac{\zeta_{26}^{6}}{46656}, \zeta_{49}=\frac{29 \zeta_{26}^{4} \zeta_{28}}{447120}, \zeta_{48}=\frac{289 \zeta_{26}^{2} \zeta_{28}^{2}}{1142640}, \\
& \zeta_{64}=\frac{5 \zeta_{26}^{3}}{216}, \zeta_{47}=\frac{39949 \zeta_{28}^{3}}{49276350}, \zeta_{27}=\frac{147 \zeta_{28}^{2}}{2645 \zeta_{26}^{2}}, \\
& \zeta_{67}=-\frac{5 \zeta_{26}^{2}}{36}, \zeta_{63}=\frac{\zeta_{26} \zeta_{28}}{92}, \zeta_{66}=-\frac{\zeta_{28}}{3}, \\
& \zeta_{25}=\frac{98 \zeta_{28}}{115 \zeta_{26}}, \zeta_{56}=-\frac{42 \zeta_{28}}{115 \zeta_{26}^{2}}, \zeta_{46}=\frac{655669 \zeta_{28}^{4}}{755570700 \zeta_{26}^{2}}, \\
& \zeta_{53}=-\frac{1764 \zeta_{28}^{2}}{2645 \zeta_{26}^{4}}, \zeta_{60}=\frac{1080}{\zeta_{26}^{3}}, \zeta_{69}=-\frac{3 \zeta_{26}}{2}, \zeta_{65}=-\frac{49 \zeta_{28}^{2}}{2645 \zeta_{26}^{2}}, \\
& \zeta_{30}=\frac{15092 \zeta_{28}^{3}}{912525 \zeta_{26}^{3}}, \zeta_{39}=-\nu^{2}+\frac{279936 \nu^{2}}{\zeta_{26}^{7}}+\frac{6391462 \zeta_{28}^{5}}{2413628625 \zeta_{26}^{5}},
\end{aligned}
$$




$$
\begin{aligned}
\zeta_{34} & =-\frac{41503 \zeta_{28}^{4}}{4197615 \zeta_{26}^{4}}, \zeta_{68}=\frac{13 \zeta_{28}}{115 \zeta_{26}}, \zeta_{58}=-\frac{28728 \zeta_{28}^{2}}{2645 \zeta_{26}^{5}}, \\
\zeta_{45} & =-\zeta_{1}\left(\mu^{2}+\nu^{2}\right)+\frac{\mu^{2} \zeta_{26}}{6}+\frac{46656 \nu^{2}}{\zeta_{26}^{6}}+\frac{1203587 \zeta_{28}^{5}}{1448177175 \zeta_{26}^{4}}, \\
\zeta_{62} & =\frac{107 \zeta_{28}^{2}}{15870 \zeta_{26}}, \zeta_{61}=\frac{2401 \zeta_{28}^{3}}{912525 \zeta_{26}^{3}}, \zeta_{59}=\frac{4536 \zeta_{28}}{23 \zeta_{26}^{4}}, \\
\zeta_{51} & =\frac{3 \zeta_{28}\left(279936 \nu^{2}+\mu^{2} \zeta_{26}^{7}\right)}{115 \zeta_{26}^{8}}-\zeta_{0}\left(\mu^{2}+\nu^{2}\right)+\frac{35153041 \zeta_{28}^{6}}{832701875625 \zeta_{26}^{6}} .
\end{aligned}
$$

Substituting Eq. (14) and Eq. (15) into Eq. (6), the 6-rogue wave solutions for Eq. (1) can be written as

$$
u=2 \Theta_{0}\left(\frac{\xi_{v v}}{\xi}-\frac{\xi_{v}^{2}}{\xi^{2}}\right)
$$

where $\xi$ satisfies Eq. (14) and Eq. (15), $\zeta_{26}$ and $\zeta_{28}$ are unrestricted. Dynamics features of 6-rogue wave solutions are shown in Figs. 7-10 when $(\mu, \nu)$ selects different values, we can see that sixrogue waves break apart and form a set of six 1-rogue waves in Figs. 7-10.
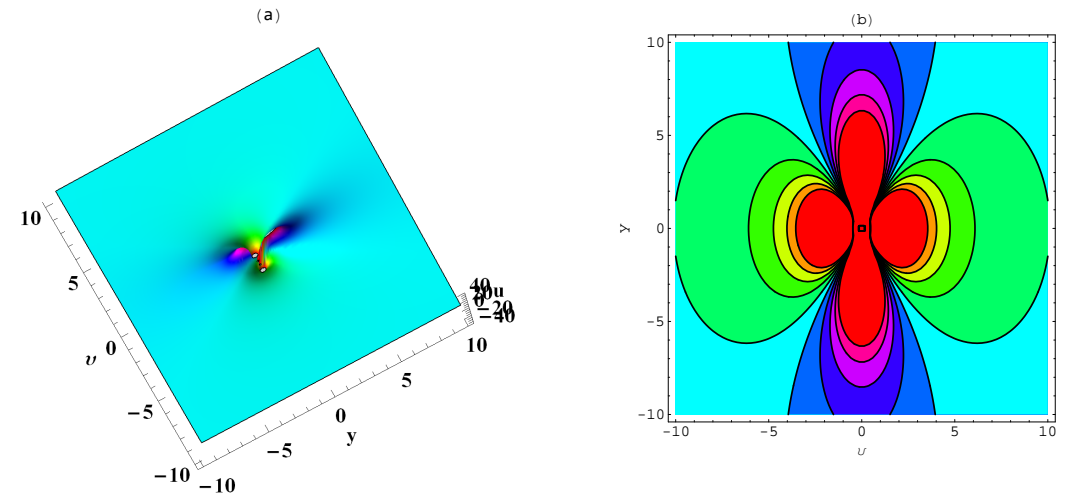

Fig. 7. Rogue wave (16) with $\mu=\nu=0, \Theta_{0}=1, \zeta_{0}=\zeta_{1}=\zeta_{28}=1$, $\zeta_{26}=2$, (a) 3D graphic, (b) contour plot.
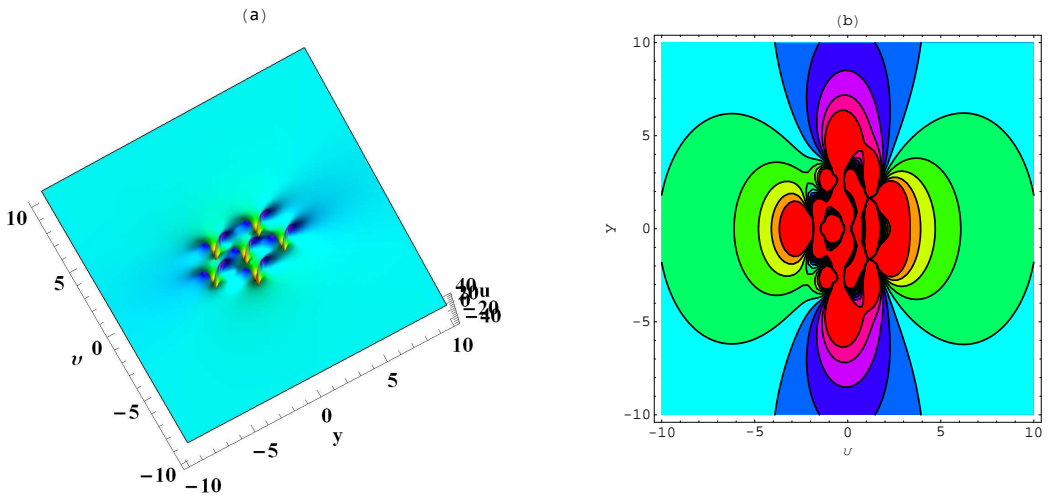
Fig. 8. Rogue wave (16) with $\mu=10, \nu=0, \Theta_{0}=1, \zeta_{0}=\zeta_{1}=\zeta_{28}=1$, $\zeta_{26}=2$, (a) 3D graphic, (b) contour plot.
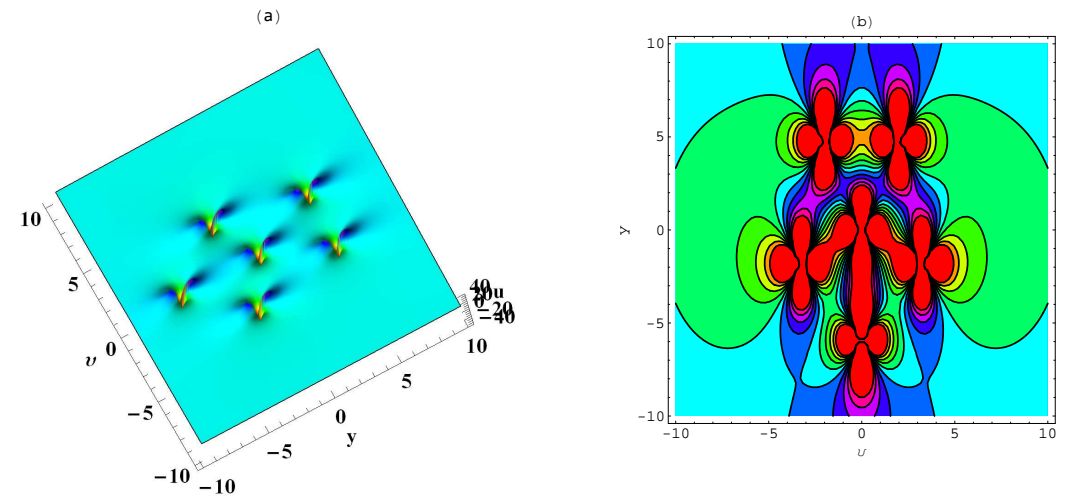

Fig. 9. Rogue wave (16) with $\mu=0, \nu=10, \Theta_{0}=1, \zeta_{0}=\zeta_{1}=\zeta_{28}=1$, $\zeta_{26}=2$, (a) 3D graphic, (b) contour plot.
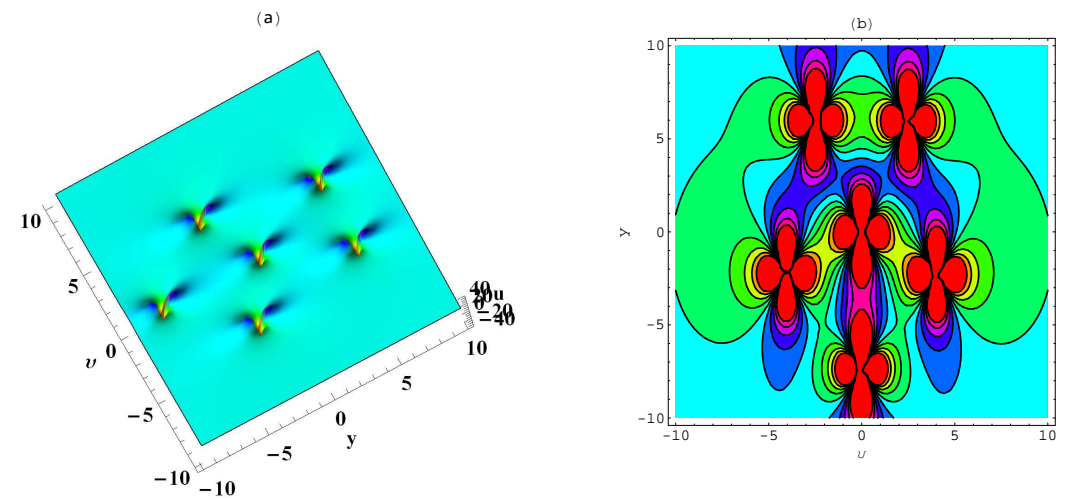

Fig. 10. Rogue wave (16) with $\mu=\nu=30, \Theta_{0}=1, \zeta_{0}=\zeta_{1}=\zeta_{28}=1$, $\zeta_{26}=2$, (a) 3D graphic, (b) contour plot.

\section{Conclusion}

In the paper, a variable-coefficient symbolic computation approach is proposed. The main difference between this method and the previous one in Ref. $[15]$ is that we replace the traveling wave transformation with the non-traveling wave transformation, making it suitable for solving the multiple rogue wave solution of the nonlinear system with variable coefficients. This change has not been seen in other literatures. Applied the vcsca to the $(2+1)$-dimensional 
vcKP equation, the 1-rogue wave solutions, 3-rogue wave solutions and 6-rogue wave solutions are present. By setting different values of $(\mu, \nu)$, their dynamics features are displayed in Figs. 1-10. All the obtained solutions have been verified to be correct.

Substituting $v=x-\omega(t)$ in rogue wave solution (10), we have

$$
u(x, y, z, t)=\frac{4 \Theta_{0}\left[-\left[\mu+\frac{3 \int \beta(t) d t}{\zeta_{0}}-x\right]^{2}+\zeta_{1}(y-\nu)^{2}+\zeta_{0}\right]}{\left[\left[\mu+\frac{3 \int \beta(t) d t}{\zeta_{0}}-x\right]^{2}+\zeta_{1}(y-\nu)^{2}+\zeta_{0}\right]^{2}} .
$$

When variable-coefficient $\beta(t)$ chooses different function, the rogue wave (17) shows different dynamics features in Fig. 11.
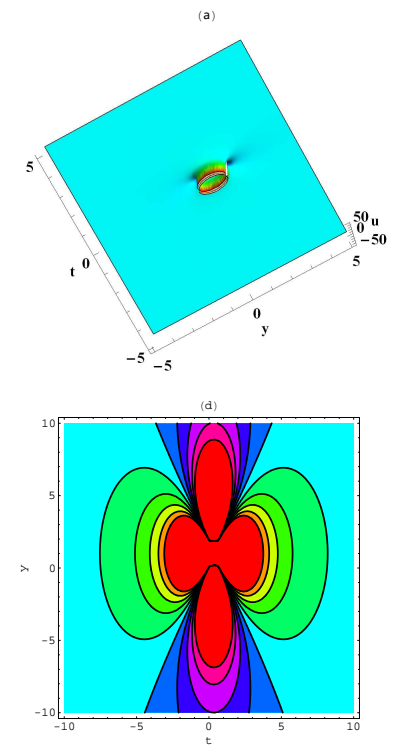
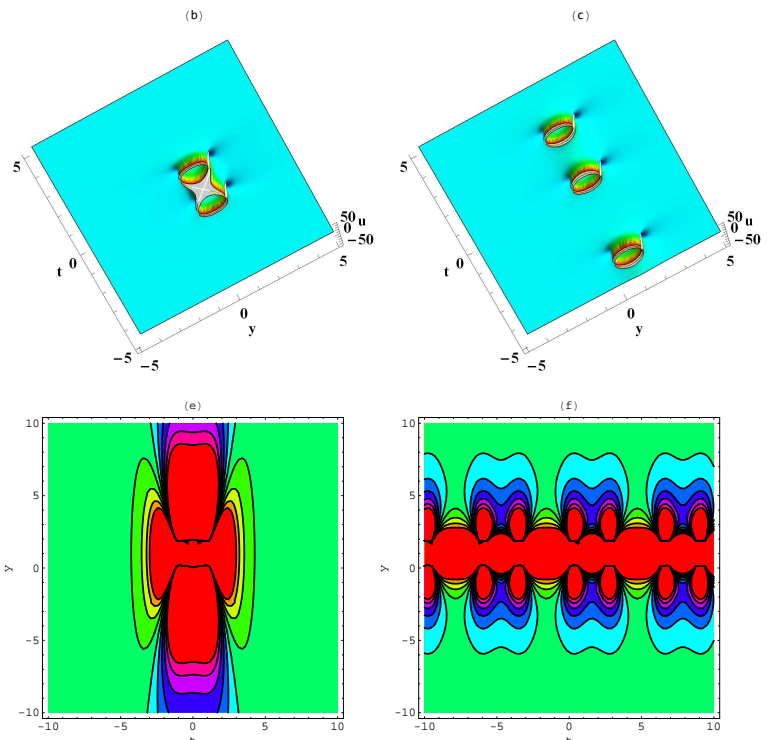

Fig. 11. Rogue wave (17) with $\mu=\nu=\Theta_{0}=1, x=0, \zeta_{0}=-1, \zeta_{1}=2$, $\beta(t)=1$ in (a) (d), $\beta(t)=t$ in (b) (e) and $\beta(t)=\cos t$ in (c) (f).

In addition to this $(2+1)$-dimensional vcKP equation, this vcsca can also be applied to the (3+1)-dimensional generalized KP equation with variable coefficients [19], the generalized $(3+1)$-dimensional variable-coefficient nonlinear-wave equation [20] based on the symbolic computation [21-26].

\section{Compliance with ethical standards}

Conflict of interests The authors declare that there is no conflict of interests regarding the publication of this article.

Ethical standard The authors state that this research complies with ethical standards. This research does not involve either human participants or animals. 


\section{References}

1. Wang, Y.Y., Zhang, J.F.: Variable-coefficient KP equation and solitonic solution for twotemperature ions in dusty plasma. Phys. Lett. A., 352(1), 155-162 (2006).

2. Yao, Z.Z., Zhang, C.Y., et al.: Wronskian and grammian determinant solutions for a variable-coefficient Kadomtsev-Petviashvili equation. Commun. Theor. Phys., 49(5), 11251128 (2008).

3. Wu, J.P.: Bilinear Bäcklund transformation for a variable-coefficient KadomtsevPetviashvili equation. Chin. Phys.s Lett., 28(6), 060207 (2011).

4. Liu, J.G., Zhu,W.H., Zhou, L.: Breather wave solutions for the Kadomtsev-Petviashvili equation with variable coefficients in a fluid based on the variable-coefficient three-wave approach. Math. Method. Appl. Sci., DOI: 10.1002/mma.5899 (2019).

5. Jia, X.Y., Tian, B., Du, Z., Sun, Y., Liu, L.: Lump and rogue waves for the variablecoefficient Kadomtsev-Petviashvili equation in a fluid. Mod. Phys. Lett. B., 32(10), 1850086 (2018)

6. Liu, J.G., Zhu,W.H., Zhou, L.: Interaction Solutions for Kadomtsev-Petviashvili Equation with Variable Coefficients. Commun. Theor. Phys., 71, 793-797 (2019).

7. Grimshaw, R., Pelinovsky, E., Taipova, T., Sergeeva, A.: Rogue internal waves in the ocean: Long wave model. Eur. Phys. J. Spec. Top., 185, 195-208 (2010).

8. Zuo, D.W., Gao, Y.T., Xue, L., Feng, Y.J., Sun, Y.H.: Rogue waves for the generalized nonlinear Schrödinger-Maxwell- Bloch system in optical-fiber communication. Appl. Math. Lett., 40, 78-83 (2015).

9. He, J.S., Charalampidis, E.G., Kevrekidis, P.G., Frantzeskakis, D.J.: Rogue waves in nonlinear Schrödinger models with variable coefficients: Application to Bose-Einstein condensates. Phys. Lett. A, 378(5-6), 577-583 (2014).

10. Li, B.Q., Ma, Y.L.: Rogue waves for the optical fiber system with variable coefficients. Optik, 158, 177-184 (2018).

11. Ankiewicz, A., Akhmediev, N.: Rogue wave-type solutions of the mKdV equation and their relation to known NLSE rogue wave solutions. Nonlinear Dyn., 91(3), 1931-1938 (2018).

12. Su, J.J., Gao, Y.T., Ding, C.C.: Darboux transformations and rogue wave solutions of a generalized AB system for the geophysical flows. Appl. Math. Lett., 88, 201-208 (2019).

13. Wang, X.B., Zhang, T.T., Dong, M.J.: Dynamics of the breathers and rogue waves in the higher-order nonlinear Schrödinger equation. Appl. Math. Lett., 86, 298-304 (2018).

14. Clarkson, P.A., Dowie, E.: Rational solutions of the Boussinesq equation and applications to rogue waves. Trans. Math. Appl., 1(1), tnx003 (2017).

15. Zha,Q.L.: A symbolic computation approach to constructing rogue waves with a controllable center in the nonlinear systems. Comput. Math. Appl., 75(9), 3331-3342 (2018).

16. Liu, W.H., Zhang, Y.F.: Multiple rogue wave solutions of the $(3+1)$-dimensional Kadomtsev-Petviashvili-Boussinesq equation. Z. Angew. Math. Phys., 70, 112 (2019).

17. Zhao, Z.L., He, L.C., Gao, Y.B.: Rogue Wave and Multiple Lump Solutions of the $(2+1)-$ Dimensional Benjamin-Ono Equation in Fluid Mechanics. Complexity,, 2019, 8249635 (2019).

18. Liu, W.H., Zhang, Y.F.: Multiple rogue wave solutions for a $(3+1)$-dimensional Hirota bilinear equation. Appl. Math. Lett., 98, 184-190 (2019).

19. Liu, J.G., Ye, Q.: Stripe solitons and lump solutions for a generalized KadomtsevPetviashvili equation with variable coefficients in fluid mechanics. Nonlinear Dyn., 96(1), 23-29 (2019)

20. Deng, G.F., Gao, Y.T.: Integrability, solitons, periodic and travelling waves of a generalized $(3+1)$-dimensional variable-coefficient nonlinear-wave equation in liquid with gas bubbles. Eur. Phys. J. Plus., 132(6), 255-271 (2017)

21. Gaillard, P.: Rational solutions to the KPI equation and multi rogue waves. Ann. Phys., 367, 1-5 (2016).

22. Yin, Y.H., Ma, W.X., Liu, J.G., Lü, X., Diversity of exact solutions to a $(3+1)-$ dimensional nonlinear evolution equation and its reduction, Comput. Math. Appl., 76, 1275-1283 (2018).

23. Lü, X., Lin, F.H., Qi, F.H.: Analytical study on a two-dimensional Korteweg-de Vries model with bilinear representation, Bäcklund transformation and soliton solutions. Appl. Math. Model., 39, 3221-3226 (2015) 
24. Xu, G.Q., Wazwaz, A.M.: Characteristics of integrability, bidirectional solitons and localized solutions for a $(3+1)$-dimensional generalized breaking soliton equation. Nonlinear Dyn., 96, 1989-2000 (2019).

25. Li, Y.Z., Liu, J.G.: New periodic solitary wave solutions for the new $(2+1)$-dimensional Korteweg-de Vries equation. Nonlinear Dyn., 91(1), 497-504 (2018).

26. Lan, Z.Z., Su, J.J.: Solitary and rogue waves with controllable backgrounds for the non-autonomous generalized AB system. Nonlinear Dyn., 96, 2535-2546 (2019) 